\title{
Off-chip beam steering with a one-dimensional optical phased array on silicon-on-insulator
}

\author{
Karel Van Acoleyen, ${ }^{1, *}$ Wim Bogaerts, ${ }^{1}$ Jana Jágerská, ${ }^{2}$ Nicolas Le Thomas, ${ }^{2}$ \\ Romuald Houdré, ${ }^{2}$ and Roel Baets ${ }^{1}$ \\ ${ }^{1}$ Photonics Research Group, Ghent University-Interuniversity MicroElectronics Center, Sint-Pietersnieuwstraat 41 , \\ B-9000 Ghent, Belgium \\ ${ }^{2}$ Institut de Photonique et d'Electronique Quantiques, Ecole Polytechnique Fédérale de Lausanne (EPFL), \\ Station 3, CH-1015 Lausanne, Switzerland \\ *Corresponding author: karel.vanacoleyen@intec.ugent.be
}

Received February 2, 2009; revised March 31, 2009; accepted April 3, 2009; posted April 8, 2009 (Doc. ID 107101); published April 30, 2009

Optical phased arrays are versatile components enabling rapid and precise beam steering. An integrated approach is followed in which a 1D optical phased array is fabricated on silicon-on-insulator. The optical phased array consists of 16 parallel grating couplers spaced $2 \mu \mathrm{m}$ apart. Steering in one direction is done thermo-optically by means of a titanium electrode on top of the structure using the phased array principle, while steering in the other direction is accomplished by wavelength tuning. At a wavelength of $1550 \mathrm{~nm}$, continuous thermo-optical steering of $2.3^{\circ}$ and wavelength steering of $14.1^{\circ}$ is reported. (C) 2009 Optical Society of America

OCIS codes: $050.0050,130.3120$.

Optical phased arrays (OPAs) allow very stable, rapid, and precise beam steering without mechanical motion, making them robust and insensitive to external constraints such as acceleration. Their operating principle is the same as the operating principle of microwave arrays [1], where a beam direction is controlled by tuning the phase relationship between arrays of transmitters. A lot of attention has been paid to liquid-crystal optical phased arrays (LC-OPAs) $[2,3]$. Such arrays actually act as a programmable blazed grating and as passive components they do not generate the steered beam.

In this Letter, an integrated approach on siliconon-insulator (SOI) is taken where the phase differences are introduced on a photonic integrated circuit and the light is subsequently coupled off chip. Phase control is done here using the thermo-optic effect in silicon. Silicon has a relatively large thermo-optic coefficient being $\partial n / \partial T=1.86 \times 10^{-4} \mathrm{~K}^{-1}$ at a wavelength of $1550 \mathrm{~nm}$ resulting in an easy, low-cost, and stable way of achieving phase modulation [4].

In [5] a similar approach is used with an AlGaAs integrated waveguide array. A continuous deflection over an angle of $0.41^{\circ}$ at a wavelength of $900 \mathrm{~nm}$ with a FWHM divergence of $0.09^{\circ}$ is reported. The phase modulation was done electro-optically with indium tin oxide/AlGaAs Schottky junctions. The array spacing is much larger than the spacing achievable with the present fabrication technologies on SOI, making the latter attractive for beam steering.

A schematic of the component can be found in Fig. 1. The component was fabricated on SOI with an oxide thickness of $2 \mu \mathrm{m}$ and a silicon thickness of 220 $\mathrm{nm}$ using standard complementary metal-oxide semiconductor (CMOS) processes in the Interuniversity MicroElectronics Center (IMEC) [6]. Two etching steps are used: one of $220 \mathrm{~nm}$ for etching the waveguides and the multimode interference (MMI) splitters and the second of $70 \mathrm{~nm}$ to etch the grating couplers. The structure is excited with transverseelectric (TE) polarized light through a lensed fiber via the facet of a 3- $\mu \mathrm{m}$-wide integrated access waveguide. This waveguide is tapered to a width of 500 $\mathrm{nm}$ and then split with a MMI-splitter tree into sixteen $800 \mathrm{~nm}$ wide parallel waveguides, spaced $2 \mu \mathrm{m}$ apart. On top of each waveguide a second-order diffraction grating intended for light outcoupling is shallow etched, characterized by a period of $630 \mathrm{~nm}$ and a duty cycle of 0.5 for efficient outcoupling of the TE polarized light. The grating coupler consists of 50 periods. After approximately 35 periods the measured outcoupled light has decayed by a factor of $1 / e^{2}$. Since transverse-magnetic (TM) polarized light will not be coupled out efficiently by the grating couplers owing to the difference in effective index, the component is highly polarization dependent. Afterward a benzocyclobutene (BCB) layer of approximately $1 \mu \mathrm{m}$ is spun on top and a titanium electrode is sputtered with a thickness of approximately 100 $\mathrm{nm}$. While the BCB does not allow for a very efficient heat transfer to the optical waveguide, this technique requires a minimum of processing to demonstrate the beam steering effect.

To determine the properties of the off-chip radiated field, the intensity of the far field needs to be mea-

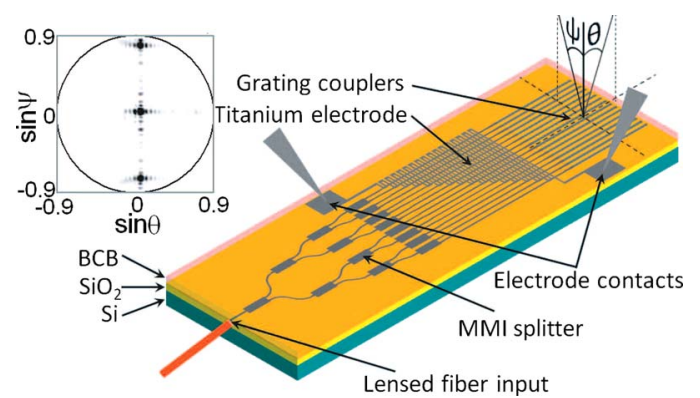

Fig. 1. (Color online) Schematic of the beam steering component. The inset shows the far-field image. 
sured in all the directions of emission. We have used a Fourier-space imaging technique developed recently at Ecole Polytechnique Fédérale de Lausanne (EPFL) [7]. Such a convenient, accurate, and fast approach consists in imaging the far field of the integrated component directly on the backfocal plane of a high-NA microscope objective $(\mathrm{NA}=0.9)$. In this plane, one point corresponds to a unique direction of off-chip emission and can be parameterized by $(\sin \theta$, $\sin \psi)$ as shown in the inset of Fig. 1. The parameters $\theta$ and $\psi$ are defined as the outcoupling angles in the longitudinal plane (i.e., along the waveguide axis) and transverse plane (i.e., perpendicular to the waveguide axis) with respect to the normal of the sample surface.

First steering along the $\theta$ angle by wavelength tuning is examined. The outcoupling angle $\theta$ is governed by the grating equation and can be expressed by

$$
\sin \theta=\frac{\Lambda n_{\mathrm{eff}}-\lambda_{0}}{n_{\mathrm{ct}} \Lambda},
$$

with $\Lambda$ being the period of the grating, $\lambda_{0}$ the freespace wavelength, $n_{\text {eff }}$ the effective index of the guided mode (equal to 2.496 at $\lambda_{0}=1550 \mathrm{~nm}$ ), and $n_{\mathrm{ct}}$ the refractive index of the background, which is air in this case. With $n_{\text {eff }}$ being a function of temperature and the wavelength, there are two ways of steering the beam along the $\theta$ angle: temperature or wavelength tuning. The latter approach is taken here. The result of wavelength tuning can be seen in Fig. 2 in which normalized far-field patterns along the $\theta$ angle are shown for three different wavelengths. However, the outcoupling efficiency of the grating couplers changes with wavelength as shown in the inset of Fig. 2, making large angle steering in this way not very attractive. The efficiency drops from $34 \%$ at $1500 \mathrm{~nm}$ to $25 \%$ at $1600 \mathrm{~nm}$ with a dip of $14 \%$ near $\lambda=1565 \mathrm{~nm}$, which corresponds to the vertical outcoupling angle $\theta=0^{\circ}$. In that case the second-order reflection back into the waveguide reduces the outcoupling efficiency. For wavelengths of 1500, 1550, and $1600 \mathrm{~nm}$ steering angles of $9.4^{\circ}, 2.0^{\circ}$, and $-4.7^{\circ}$

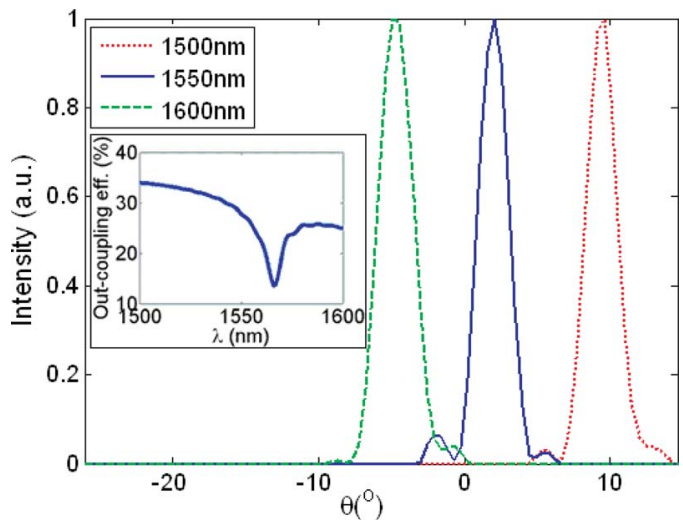

Fig. 2. (Color online) Normalized far-field pattern along the $\theta$ angle for wavelengths of 1500, 1550, and $1600 \mathrm{~nm}$. The inset shows the simulated outcoupling efficiency of one grating coupler. with FWHM divergences of $2.4^{\circ}, 2.5^{\circ}$, and $2.8^{\circ}$, respectively, are observed.

Steering along the $\psi$ angle (see Fig. 1) is based on the phased array principle. The lateral outcoupling angle $\psi$ of a phased array with spacing $d$ of the grating couplers is given by

$$
\sin \psi=\frac{\lambda_{0} \phi}{2 \pi d}
$$

with $\phi$ being the uniform phase difference between the grating couplers. In Fig. 3 the far-field profile of a reference array of 16 grating couplers without the heater electrode and the corresponding long waveguide region is shown at a wavelength of $1550 \mathrm{~nm}$. The measurement result is fit to a simulation of an array of Gaussian beams in one dimension. When the individual Gaussians are modeled with a 1/e mode width of $680 \mathrm{~nm}$, we can see a nice agreement with the measurement. This mode width corresponds to the average 1/e mode width just above the grating coupler and determines the envelope of the far-field pattern. The dashed curve shows the simulated far field of one such Gaussian. The beam width is inversely proportional to the total aperture size of the array and the cosine of the steering angle $\psi$,

$$
\Delta \psi_{\mathrm{FWHM}} \approx \frac{0.886 \lambda_{0}}{N d \cos \psi},
$$

with $N$ being the number of array elements and $d$ the spacing between the elements [1]. The measured FWHM is $2.7^{\circ}$ compared with a simulated FWHM of $2.4^{\circ}$. The second-order peaks of the grating can be found at an angle of $\psi=\arcsin \left(\lambda_{0} / d\right)=50.8^{\circ}$. This angle can be increased by reducing the separation between the individual grating couplers. Alternatively, the intensity of the second-order peaks can be reduced by using more directive elements of the array, which would result in a constriction of the envelope of the far-field pattern. The sidelobes can be suppressed by using a nonuniform excitation of the ar-

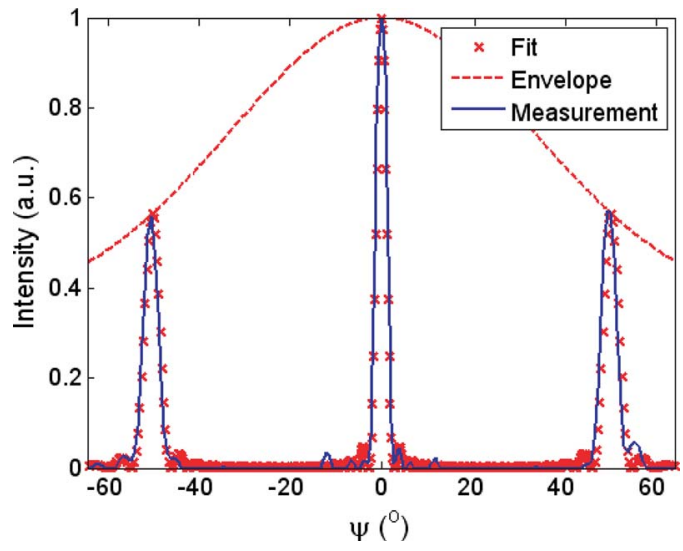

Fig. 3. (Color online) Normalized far-field pattern along the $\psi$ angle of a reference array of 16 grating couplers at $1550 \mathrm{~nm}$ without the heater electrode. The crosses show a simulation fit. The dashed curve shows the simulated farfield pattern of one grating coupler. 
ray, i.e., by apodization of the light intensity coupled into the individual grating couplers.

Steering can be performed by temperature tuning. When a current is flowing through the titanium electrode, a linear phase ramp is imposed on the array of waveguides owing to the Joule heating and the specific shape of the electrode. The far-field pattern of the device under investigation is shown in inset (a) of Fig. 4. The peaks are no longer centered at $\psi=0^{\circ}$ owing to a fixed phase shift present in the waveguides. These phase shifters consist of a slight increase in the waveguide width resulting in a different effective index of the mode and were present for static beam deflection measurements. The long waveguide lines together with the fixed phase shifters introduce random phase errors and result in unwanted sidelobe levels. This random phase noise can be modeled as Gaussian and, as the number of elements increases, the effect of the phase errors becomes less significant as has been shown in [8]. The main peak (at $\psi_{0}=20^{\circ}$ ), however, remains clearly visible and can be steered dynamically by current injection. The percentage of power in this main beam was measured to be $42.2 \%$, compared with a theoretical percentage of $44.4 \%$. The results of the steering with current injection by increasing the current from 0 to $3.95 \mathrm{~mA}$ can be found in inset (b) of Fig. 4. The far-field pattern along the $\psi$ angle for currents of 0 and $3.95 \mathrm{~mA}$ together with a sinc fit of the latter is shown in Fig. 4. Higher currents were not used owing to practical limitations

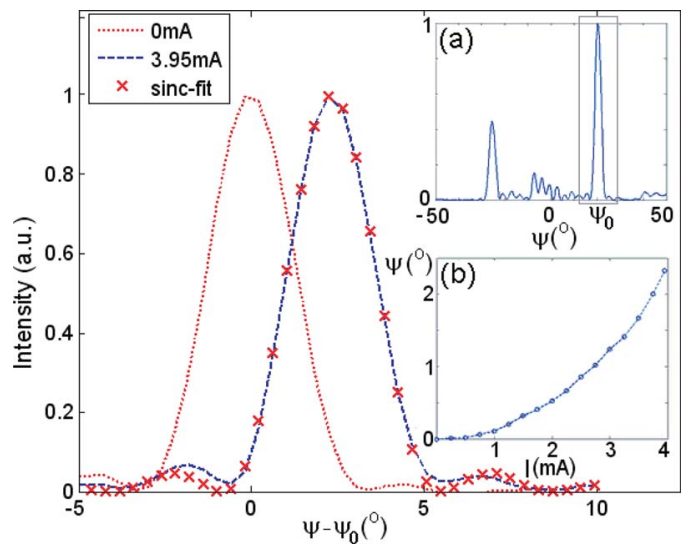

Fig. 4. (Color online) Normalized far-field pattern along the $\psi$ angle of an array of 16 grating couplers with injection currents of 0 and $3.95 \mathrm{~mA}$. The peak at a current of $0 \mathrm{~mA}$ is the detail of the main peak around $20^{\circ}$ shown in inset (a). The crosses show a sinc fit. Inset (b) shows the steering angle $\psi$ as a function of the injected current. and owing to the possibility of burning the resistor electrode.

The shift at a current of $3.95 \mathrm{~mA}$ was $2.3^{\circ}$, which implies a phase difference of $\phi=0.105 \pi$ between the grating couplers. The electrode thus imposes this phase shift at the first waveguide, twice this phase shift at the second waveguide, etc. The total phase shift induced by the electrode is $\Phi_{\text {tot }}=(1+2+\cdots$ $+16) 0.105 \pi=14.28 \pi$. The measured resistance of the electrode was approximately $15 \mathrm{k} \Omega$ of which approximately half was used for the phase shift such that the power needed for a $\pi$ phase shift is given by $P_{\pi}$ $=8.2 \mathrm{~mW}$.

In conclusion, an OPA was fabricated on SOI. Beam steering was done thermo-optically by means of Joule heating. In this way, steering over an angle of $2.3^{\circ}$ at a wavelength of $1550 \mathrm{~nm}$ was demonstrated. By using the proper heating electrode layout, addressing issues can be resolved, since only one electrode is needed to achieve steering. By placing a mirrored electrode, steering could be achieved in both senses of the transverse direction with only two electrodes.

The authors acknowledge support from the European projects ePIXnet (IST-004525), the European Cooperation in the field of Scientific and Technical Research (COST) MP0702 action, and the Swiss National Centre of Competence in Research-Quantum Photonics. K. Van Acoleyen acknowledges the Research Foundation-Flanders (FWO) for a research grant. W. Bogaerts acknowledges the FWO for a postdoctoral fellowship.

\section{References}

1. M. I. Skolnik, Introduction to Radar Systems (McGrawHill, 1962).

2. P. F. McManamon, T. A. Dorschner, D. L. Corkum, L. J. Friedman, D. S. Hobbs, M. Holz, S. Liberman, H. Q. Nguyen, D. P. Resler, R. C. Sharp, and E. A. Watson, Proc. IEEE 84, 268 (1996).

3. L. Shi, P. F. McManamon, and P. J. Bos, J. Appl. Phys. 104, 033109 (2008).

4. V. M. N. Passaro, F. Magno, and A. V. Tsarev, Opt. Express 13, 3429 (2005).

5. F. Vasey, F. K. Reinhart, R. Houdré, and J. M. Stauffer, Appl. Opt. 32, 3220 (1993).

6. ePIXfab, the silicon photonics platform, http:// www.epixfab.eu/.

7. N. Le Thomas, R. Houdré, M. V. Kotlyar, D. O'Brien, and T. F. Krauss, J. Opt. Soc. Am. B 24, 2964 (2007).

8. A. Polishuk and S. Arnon, Opt. Eng. 42, 2015 (2003). 